\title{
REFERENCES
}

1. E. Steinitz, J. Reine Angew. Math. vol. 143 (1913) pp. 128-275.

2. W. Fenchel, Über Krilmmung und Windung geschlossener Raumkurven, Math. Ann. vol. 101 (1929) pp. 238-252.

3. L. N. H. Bunt, Bijdrage tot de theorie der convexe puntverzamelingen, Amsterdam, 1934.

UNIVERSITY OF STOCKHOLM

\section{ON APPELL POLYNOMIALS}

R. S. VARMA

1. Sheffer [1] ${ }^{1}$ and Thorne [2] have, in their papers, given Stieltjes integral characterization to Appell sets of polynomials, defined by

$$
P_{n}^{\prime}(x)=P_{n-1}(x) .
$$

The generating function $A(t)$ of an Appell set $\left[P_{n}(x)\right]$ is given by

$$
e^{t x} A(t)=\sum_{n=0}^{\infty} t^{n} P_{n}(x) .
$$

According to Sheffer, a polynomial $P_{n}(x)$ is an Appell set if and only if there is a function $\beta(x)$ of bounded variation on $(0, \infty)$ with the following properties:

(i) The moment constants $b_{n}=\int_{0}^{\infty} x^{n} d \beta(x)$ all exist.

(ii) $b_{0} \neq 0$.

(iii) For $n=0,1,2, \cdots$,

$$
P_{n}(x)=\int_{0}^{\infty} \frac{(x+t)^{n}}{n !} d \beta(t) .
$$

2. More generally (Sheffer, p. 741) the function $(x+t)^{n} / n$ ! can be replaced by $Q_{n}(x+t)$ where $\left\{Q_{n}(x)\right\}$ is any Appell set. Now constants $\left\{q_{k}\right\}$ exist such that

$$
Q_{n}(x+t)=q_{0} \frac{(x+t)^{n}}{n !}+q_{1} \frac{(x+t)^{n-1}}{(n-1) !}+\cdots+q_{n} .
$$

This suggests the following generalization: Let $\left\{\delta_{n}(t)\right\}$ be any sequence of functions for which the integrals

Received by the editors May 28, 1949 and, in revised form, July 14, 1950.

${ }^{1}$ Numbers in brackets refer to the references cited at the end of the paper. 
(a)

$$
I_{n, r}=\int_{0}^{\infty} \delta_{n}(t) t^{r} d \beta(t)
$$

exist, $n, r=0,1, \cdots$, with $I_{0,0} \neq 0$, and define

(b) $\quad K_{n}(x, t)=\delta_{0}(t) \frac{(x+t)^{n}}{n !}+\delta_{1}(t) \frac{(x+t)^{n-1}}{(n-1) !}+\cdots+\delta_{n}(t)$.

Then

$$
P_{n}(x)=\int_{0}^{\infty} K_{n}(x, t) d \beta(t)
$$

is an Appell set.

As a particular instance of this result, let

$$
{ }_{3} F_{2}(\alpha, a, b ; c, d ; u)=\sum_{j=0}^{\infty} \frac{(\alpha, j)(a, j)(b, j)}{(c, j)(d, j)} \frac{u^{j}}{j !} .
$$

For $n=0,1, \cdots$, it is readily found that

$$
\frac{x^{n}}{n !}{ }_{3} F_{2}(-n, a, b ; c, d ;-t / x)=K_{n}(x, t)
$$

with

$$
\delta_{r}(t)=\frac{t^{r}}{r !}\left\{\alpha_{r}-C_{r, 1} \alpha_{r-1}+\cdots+(-)^{r} C_{r, r} \alpha_{0}\right\}
$$

where

$$
\alpha_{0}=1, \quad \alpha_{i}=\frac{(a, j-1)(b, j-1)}{(c, j-1)(d, j-1)}
$$

Hence

$$
\begin{aligned}
P_{n}^{*}(x) & =\int_{0}^{\infty} \frac{x^{n}}{n !}{ }_{3} F_{2}(-n, a, b ; c, d ;-t / x) d \beta(t) \\
& =\frac{1}{n !} \sum_{r=0}^{\infty}(-) r \frac{(-n, r)(a, r)(b, r)}{(c, r)(d, r)} \frac{\beta_{r}}{r !} x^{n-r}
\end{aligned}
$$

is an Appell set. Here $\left\{\beta_{r}\right\}$ is the set of moment constants and $\beta(t)$ is a function of the type considered by Sheffer.

On account of the identity

$$
{ }_{2} F_{1}(-n, a ; a ;-u)=(1+u)^{n},
$$


the function defined by (1) reduces to Appell polynomials given by (iii) above, when we take $a=c$ and $b=d$.

3. The generating function $A^{*}(u)$ of the polynomials given by (1) will be

$$
\begin{aligned}
\sum_{n=0}^{\infty} P_{n}^{*}(x) u^{n} & =\sum_{n=0}^{\infty}\left\{\frac{(x u)^{n}}{n !}\right\} \int_{0}^{\infty}{ }_{3} F_{2}(-n, a, b ; c, d ;-t / x) d \beta(t) \\
& =\int_{0}^{\infty} \sum_{n=0}^{\infty} \frac{(x u)^{n}}{n !}{ }_{3} F_{2}(-n, a, b ; c, d ;-t / x) d \beta(t)
\end{aligned}
$$

on interchanging the order of integration and summation. Summing up the infinite series by means of a formula due to Rice [3], we have

$$
\begin{aligned}
e^{u x} A^{*}(u) & =\sum_{n=0}^{\infty} P_{n}(x) u^{n} \\
& =e^{u x} \int_{0}^{\infty}{ }_{2} F_{2}(a, b ; c, d ; u t) d \beta(t),
\end{aligned}
$$

giving that

$$
A^{*}(u)=\int_{0}^{\infty}{ }_{2} F_{2}(a, b ; c, d ; u t) d \beta(t)
$$

4. In the case when $b=d$, the function ${ }_{2} F_{2}$ reduces to Kummer's function ${ }_{1} F_{1}$ and the formula for the generating function assumes an interesting form when we use the following integral representation for Kummer's function:

$$
{ }_{1} F_{1}(a ; c ; y)=\frac{\Gamma(c)}{\Gamma(a) \Gamma(c-a)} \int_{0}^{1} e^{x y} x^{a-1}(1-x)^{c-a-1} d x .
$$

Thus

$$
\begin{aligned}
A^{*}(u) & =\frac{\Gamma(c)}{\Gamma(a) \Gamma(c-a)} \int_{0}^{\infty}\left[\int_{0}^{1} e^{u t x} x^{a-1}(1-x)^{c-a-1} d x\right] d \beta(t) \\
& =\frac{\Gamma(c)}{\Gamma(a) \Gamma(c-a)} \int_{0}^{1} x^{a-1}(1-x)^{c-a-1}\left[\int_{0}^{\infty} e^{u t x} d \beta(t)\right] d x \\
& =\frac{\Gamma(c)}{\Gamma(a) \Gamma(c-a)} \int_{0}^{1} x^{a-1}(1-x)^{c-a-1} A(u x) d x
\end{aligned}
$$

where $A(u)$ is the generating function involved in Sheffer's result. In this way, we have the generating function for our Appell polynomials 
in terms of the generating function of the corresponding Appell polynomials given by (iii).

As an example, consider the Hermite polynomials $H_{n}(x)$ defined by

$$
e^{t x-t^{2} / 2}=\sum_{n=0}^{\infty} \frac{H_{n}(x)}{n !} t^{n}
$$

$\left\{H_{n}(x) / n !\right\}$ is an Appell set, the generating function for which is

$$
A(t)=e^{-t^{2} / 2} \text {. }
$$

It is easy to see that the generating function corresponding to polynomials contemplated in $\$ 2$ is

$$
A^{*}(u)=\frac{\Gamma(c)}{\Gamma(a) \Gamma(c-a)} \int_{0}^{1} x^{a-1}(1-x)^{c-a-1} e^{-u^{2} x^{2} / 2} d x .
$$

Expanding the exponential function and integrating term by term, we have, after some simplification, by the help of elementary results in the gamma function,

$$
A^{*}(u)={ }_{2} F_{2}\left(a / 2,(a+1) / 2 ; c / 2,(c+1) / 2 ;-u^{2} / 2\right) .
$$

The corresponding generalized Hermite polynomial will be

$$
\begin{gathered}
x^{n}-\frac{(-n, 2)(a, 2)}{(c, 2)} \frac{x^{n-2}}{2}+\frac{(-n, 4)(a, 4)}{(c, 4)} \frac{x^{n-4}}{2^{2} 2 !} \\
-\frac{(-n, 6)(a, 6)}{(c, 6)} \frac{x^{n-6}}{2^{3} 3 !}+\cdots .
\end{gathered}
$$

Finally I thank the referee for his valuable suggestions.

\section{REFERENCES}

1. I. M. Sheffer, Bull. Amer. Math. Soc. vol. 51 (1945) pp. 739-744.

2. C. J. Thorne, Amer. Math. Monthly vol. 52 (1945) pp. 191-193.

3. S. O. Rice, Duke Math. J. vol. 6 (1940) pp. 108-119.

LUCKNOW UNIVERSITY 